\title{
Acceleromyography at the Flexor Hallucis Brevis Muscle Underestimates Residual Neuromuscular Blockade
}

\author{
Yasuyuki Sugi*, Keiichi Nitahara, Kiyoshi Katori, Go Kusumoto, Kenji Shigematsu and Kazuo \\ Higa
}

Department of Anesthesiology, Fukuoka University Faculty of Medicine, 7-45-1 Nanakuma, Jonan-ku, Fukuoka 8140180, Japan

\begin{abstract}
Purpose: Recovery of the train-of-four ratio (TOFR) to $>0.9$ in the upper limb is commonly used to determine that neuromuscular function has returned to the preoperative level. It is not known whether recovery of neuromuscular function can be determined in the same way using lower limb acceleromyography. We compared measurements of recovery from neuromuscular blockade using upper limb electromyography and lower limb acceleromyography.
\end{abstract}

\begin{abstract}
Methods: Twenty-nine patients who were scheduled for elective surgery were enrolled in this study. Patients were excluded if they had neuromuscular disease or contraindications to neuromuscular blockade. General anesthesia was induced and maintained with propofol and fentanyl. Patients were monitored using electromyography at the first dorsal interosseous muscle of the upper limb and acceleromyography at the flexor hallucis brevis muscle of the lower limb. Vecuronium $0.1 \mathrm{mg} / \mathrm{kg}$ was administered for neuromuscular blockade, and the profile of the blockade was recorded, including onset time and recovery times to TOFR 0.7 and 0.9 . Results were compared between the upper and lower limbs.
\end{abstract}

Results: The first dorsal interosseous muscle of the upper limb was slower to recover to TOFR 0.7 and 0.9 than the flexor hallucis brevis muscle. When the TOFR at the flexor hallucis brevis muscle had recovered to 0.9, the TOFR at the first dorsal interosseous muscle was $0.44 \pm 0.23$.

Conclusion: Monitoring the flexor hallucis brevis muscle using acceleromyography underestimates the residual neuromuscular blockade.

Keywords: Acceleromyography, electromyography, neuromuscular blockade, neuromuscular monitoring, neuromuscular function, residual neuromuscular blockade.

\section{INTRODUCTION}

Neuromuscular monitoring of the upper limb is used to determine when swallowing and respiratory functions have returned to pre-neuromuscular blockade levels [1-5]. To prevent postoperative respiratory complications, TOFR of the upper limb should recover to $>0.9$ before extubation $[2,6-$ 10]. However, it is difficult to monitor a patient's upper limb responses to neuromuscular blockade if their arms are not accessible because they are involved with the surgery or tucked at their side. In such cases, attaching a neuromuscular monitor to the flexor hallucis brevis muscle of the lower limb is an easy and useful alternative [11-15]. Many studies have compared the recovery of upper and lower limb blockade by non-depolarizing neuromuscular blockers to TOFR $<$ 0.75 [11-15]. A recent study found that TOFR monitoring of eye muscles led to an increased risk of postoperative residual paralysis compared with TOFR monitoring of an upper limb muscle [16]. It is not known whether TOFR $>0.9$ of a lower

*Address correspondence to this author at the Department of Anesthesiology, Fukuoka University Faculty of Medicine, 7-45-1 Nanakuma, Jonan-ku, Fukuoka 814-0180, Japan; Tel: +81-92-801-1011; Fax: +81-92-865-5816; E-mail: ysugi@fukuoka-u.ac.jp limb muscle is interchangeable with that of an upper limb muscle.

Vecuronium is a steroidal nondepolarizing neuromuscular agent with an intermediate duration of action. We compared the differences in recovery from vecuronium-induced neuromuscular blockade using electromyography at the first dorsal interosseous muscle of the upper limb and acceleromyography at the flexor hallucis brevis muscle of the lower limb.

\section{METHODS}

The Ethical Committee of Fukuoka University approved the study protocol on 26 October 2005 . Written consent was obtained from all patients. Twenty-nine patients aged 18 to 70 years who were scheduled for otolaryngologic or ophthalmic surgery under general anesthesia were enrolled in the study. Patients were excluded if they were taking drugs that affect neuromuscular junctions, or had hepatic dysfunction, renal dysfunction, endocrine dysfunction, metabolic or neuromuscular disease, or a body mass index $>30 \mathrm{~kg} / \mathrm{m}^{2}$.

Diazepam $(5-10 \mathrm{mg}$ ) was administered orally as premedication. Electrocardiography, non-invasive blood pres- 
sure, and pulse oximetry monitors were attached after entering the operating room. To monitor neuromuscular blockade, electromyography electrodes (M-NMT monitor ${ }^{\mathbb{B}}, \mathrm{AS} / 3$, Datex-Ohmeda, Finland) were attached over the first dorsal interosseous muscle of the upper limb, and an acceleration transducer (TOF-Watch $\mathrm{SX}^{\mathbb{R}}$, Organon, Netherlands) was attached over the middle of the great toe. Upper and lower limb stimulation electrodes (NMT electrodes ${ }^{\circledR}$, DatexOhmeda) were attached over the ulnar nerve proximal to the wrist joint and the tibial nerve at the inferolateral aspect of the medial malleolus, respectively.

Anesthesia was induced with propofol $(1-2 \mathrm{mg} / \mathrm{kg})$ and fentanyl $(100-200 \mu \mathrm{g})$, and was maintained with a continuous propofol infusion $(4-10 \mathrm{mg} / \mathrm{kg} / \mathrm{h})$. Additional fentanyl was administered as required. After loss of consciousness, $50-\mathrm{Hz}$ tetanic stimulation was applied to the posterior tibial nerve for $5 \mathrm{~s}$ to shorten the stabilization period $[17,18]$, followed by calibration of the upper and lower limb neuromuscular monitors. Four consecutive supramaximal stimuli were then delivered to the ulnar nerve at 20 -s intervals and the posterior tibial nerve at 15 -s intervals. After a 5-min stabilization period, control values for TOFR at the first dorsal interosseous muscle and $\mathrm{T} 1 / \mathrm{T} 0$ at the flexor hallucis brevis muscle were recorded. Vecuronium $(0.1 \mathrm{mg} / \mathrm{kg})$ was administered intravenously via a dorsal vein of the hand, followed immediately by a $5-\mathrm{mL}$ bolus of normal saline. Tracheal intubation was then performed. The onset time of vecuronium was defined as the time from the end of vecuronium administration to $95 \%$ suppression of T1. Intraoperative ventilation was adjusted to an end-expiratory partial pressure of carbon dioxide of 30-40 mm Hg. Subjects were warmed with a heating blanket to ensure that the skin temperature of the upper and lower limbs that were being monitored did not drop below $32^{\circ} \mathrm{C}$. No additional vecuronium was administered during surgery. Spontaneous recovery of neuromuscular blockade was observed until the TOFR at the first dorsal interosseous muscle had recovered to 0.7 and the TOFR at the flexor hallucis brevis muscle had recovered to 0.9 . TOFRs were also recorded at the first dorsal interosseous muscle when the TOFR at the flexor hallucis brevis muscle had recovered to 0.7 and 0.9 . No muscle relaxant antagonist was used.

An average standard deviation value of 23 was used to calculate the required sample size for examining spontaneous recovery to TOFR 0.7 after $0.1 \mathrm{mg} / \mathrm{kg}$ of vecuronium [19]. If the difference in recovery times between the first dorsal interosseous muscle and the flexor hallucis brevis muscle was $20 \mathrm{~min}, 22$ cases were needed to achieve $90 \%$ detection power at a significance level of 0.05 . Recovery times at the first dorsal interosseous muscle and the flexor hallucis brevis muscle were compared using the paired t-test. A $P$ value $\leq$ 0.05 was considered statistically significant. Patient characteristics and recordings are shown as the mean \pm standard deviation with a $95 \%$ confidence interval (CI). Analyses were performed using StatView ${ }^{\circledR}$ software version 5.0 (SAS Institute Inc., Cary, NC, USA).

\section{RESULTS}

Seventeen male and 12 female patients were enrolled in this study. The patients had a mean age of $44 \pm 17$ years, height of $163 \pm 48 \mathrm{~cm}$, and weight of $65 \pm 12 \mathrm{~kg}$. The onset time of neuromuscular blockade after vecuronium administration was $137 \pm 33 \mathrm{~s}$ at the first dorsal interosseous muscle and $143 \pm 19 \mathrm{~s}$ at the flexor hallucis brevis muscle, which was not a significant difference between the two muscles. The recovery time from vecuronium administration to TOFR 0.7 and 0.9 was significantly longer at the first dorsal interosseous muscle than at the flexor hallucis brevis muscle $(P<0.05)$ (Table 1). The TOFR at the first dorsal interosseous muscle was $0.24 \pm 0.19$ (95\% CI 0.19 to 0.34$)$ when the flexor hallucis brevis muscle had recovered to TOFR 0.7, and $0.44 \pm 0.23(95 \%$ CI 0.36 to 0.53$)$ when the flexor hallucis brevis muscle had recovered to TOFR 0.9 (Table 2).

Table 1. Recovery Times at the Flexor Hallucis Brevis Muscle and the First Dorsal Interosseous Muscle after Administration of Vecuronium $0.1 \mathrm{mg} / \mathrm{kg}(n=29)$

\begin{tabular}{|c|c|c|c|}
\hline & FHBM & FDIM & FDIM - FHBM \\
\hline \hline Time to TOFR $0.7^{\mathrm{a}}(\mathrm{min})$ & $51 \pm 14^{*}$ & $69 \pm 20^{*}$ & $18(14.5-23.1)$ \\
\hline Time to TOFR $0.9^{\mathrm{b}}(\mathrm{min})$ & $62 \pm 20^{*}$ & $89 \pm 33^{*}$ & $27(19.2-35.6)$ \\
\hline
\end{tabular}

Data are presented as mean \pm standard deviation for FHBM and FDIM, (95\% CI for FDIM - FHBM).

*Significantly different between the muscles; $P<0.0001$.

a Time from administration of vecuronium $0.1 \mathrm{mg} / \mathrm{kg}$ to recovery of TOFR to 0.7

${ }^{\mathrm{b}}$ Time from administration of vecuronium $0.1 \mathrm{mg} / \mathrm{kg}$ to recovery of TOFR to 0.9 .

Confidence interval (CI), first dorsal interosseous muscle (FDIM), flexor hallucis brevis muscle (FHBM), train-of-four ratio (TOFR).

Table 2. TOFR at the First Dorsal Interosseous Muscle when TOFR was 0.7 and 0.9 at the Flexor Hallucis Brevis Muscle

\begin{tabular}{|c|c|}
\hline TOFR of the FHBM & TOFR of the FDIM \\
\hline \hline 0.7 & $0.24 \pm 0.19(95 \%$ CI; $0.19-0.34)$ \\
\hline 0.9 & $0.44 \pm 0.23(95 \%$ CI; $0.36-0.53)$ \\
\hline
\end{tabular}

Data are presented as mean \pm standard deviation $(95 \% \mathrm{CI})$.

Confidence interval (CI), first dorsal interosseous muscle (FDIM), flexor hallucis brevis muscle (FHBM), train-of-four ratio (TOFR). 


\section{DISCUSSION}

The TOFR using electromyography at the first dorsal interosseous muscle was very low at $0.44 \pm 0.23$ when the TOFR using acceleromyography at the flexor hallucis brevis muscle had recovered to 0.9 . If recovery from neuromuscular blockade has been assessed using the flexor hallucis brevis muscle, it is safer to also perform postoperative assessment of TOFR at the first dorsal interosseous muscle or the adductor pollicis muscle.

Previous studies comparing the recovery of upper and lower limb neuromuscular blockade by non-depolarizing relaxants were all limited to recovery of TOFR to $<0.75$ [11-15]. Saitoh et al. [15] compared the recovery times from $0.2 \mathrm{mg} / \mathrm{kg}$ of vecuronium at the adductor pollicis and flexor hallucis brevis muscles using acceleromyography, and reported that recovery to TOFR 0.2 and 0.6 was significantly faster at the flexor hallucis brevis muscle. Heier et al. [11] used mechanomyography at the adductor pollicis muscle and acceleromyography at the flexor hallucis brevis muscle to compare the recovery time from $0.1 \mathrm{mg} / \mathrm{kg}$ of vecuronium, and reported that recovery to TOFR 0.75 was 13 min longer at the adductor pollicis muscle. In this study, we found that recovery to TOFR 0.7 was 19 min longer in the upper limb than in the lower limb, and recovery to TOFR 0.9 was also longer in the upper limb.

There was no significant difference in the time of onset of neuromuscular blockade after vecuronium administration between the first dorsal interosseous muscle and the flexor hallucis brevis muscle. Previous studies comparing times of onset of non-depolarizing neuromuscular blockade reported faster onset at the adductor pollicis muscle than the flexor hallucis brevis muscle $[11,12]$. In this study, we did not find a significant difference in onset times between upper and lower limb muscles. This is thought to be because of differences in the muscles measured and the monitoring methods used.

Mechanomyography at the adductor pollicis muscle is the standard method for monitoring neuromuscular blockade in clinical research, and many studies confirming that recovery of TOFR to 0.7 or 0.9 is essential for safe extubation have used this technique [20-23]. In the present study, we used electromyography at the first dorsal interosseous muscle of the upper limb. This was because mechanomyography was not available at our institution, and because there is a good correlation between adductor pollicis mechanomyography measurements and first dorsal interosseous electromyography measurements during the time of recovery of TOFR to 0.7-0.9. The differences in results between the two methods decreases during the later stages of recovery, and was reported to be negligible at TOFR $>0.75$ [24].

A recent study found that TOFR monitoring of eye muscles led to an increased risk of postoperative residual paralysis compared with TOFR monitoring of an upper limb muscle [16]. This increased risk of residual paralysis may also occur with TOFR monitoring of lower limb muscles, because the lower limb muscles recover faster from neuromuscular blockade than the upper limb muscles [11-15].

Recovery from vecuronium-induced neuromuscular blockade in patients under anesthesia maintained with propo- fol and fentanyl was compared between the upper limb using electromyography at the first dorsal interosseous muscle and the lower limb using acceleromyography at the flexor hallucis brevis muscle. When the flexor hallucis brevis muscle had recovered to TOFR 0.9 , the TOFR at the first dorsal interosseous muscle was low at $0.44 \pm 0.23$.

\section{CONFLICT OF INTEREST}

The authors confirm that this article content has no conflicts of interest.

\section{ACKNOWLEDGEMENT}

None declared.

\section{REFERENCES}

[1] Murphy GS, Szokol JW, Marymont JH, Greenberg SB, Avram MJ, Vender JS. Residual neuromuscular blockade and critical respiratory events in the postanesthesia care unit. Anesth Analg 2008; 107: 130-7.

[2] Eikermann M, Blobner M, Groeben H, et al. Postoperative upper airway obstruction after recovery of the train of four ratio of the adductor pollicis muscle from neuromuscular blockade. Anesth Analg 2006; 102: 937-42.

[3] Sundman E, Witt H, Olsson R, Ekberg O, Kuylenstierna R, Eriksson LI. The incidence and mechanisms of pharyngeal and upper esophageal dysfunction in partially paralyzed humans: pharyngeal videoradiography and simultaneous manometry after atracurium. Anesthesiology 2000; 92: 977-84.

[4] Kopman AF, Yee PS, Neuman GG. Relationship of the train-offour fade ratio to clinical signs and symptoms of residual paralysis in awake volunteers. Anesthesiology 1997; 86: 765-71.

[5] Berg H, Roed J, Viby-Mogensen J, et al. Residual neuromuscular block is a risk factor for postoperative pulmonary complications. A prospective, randomised, and blinded study of postoperative pulmonary complications after atracurium, vecuronium and pancuronium. Acta Anaesthesiol Scand 1997; 41: 1095-103.

[6] Maybauer DM, Geldner G, Blobner M, et al. Incidence and duration of residual paralysis at the end of surgery after multiple administrations of cisatracurium and rocuronium. Anaesthesia 2007; 62: 12-7.

[7] Murphy GS, Szokol JW, Marymont JH, Franklin M, Avram MJ, Vender JS. Residual paralysis at the time of tracheal extubation. Anesth Analg 2005; 100:1840-5.

[8] Baillard C, Clec'h C, Catineau J, et al. Postoperative residual neuromuscular block: a survey of management. Br J Anaesth 2005; 95 : 622-6.

[9] Kopman AF, Yee PS, Neuman GG. Relationship of the train-offour fade ratio to clinical signs and symptoms of residual paralysis in awake volunteers. Anesthesiology 1997; 86: 765-71.

[10] Kopman AF, Ng J, Zank LM, Neuman GG, Yee PS. Residual postoperative paralysis. Pancuronium versus mivacurium, does it matter? Anesthesiology 1996; 85: 1253-9.

[11] Heier T, Hetland S. A comparison of train-of-four monitoring: mechanomyography at the thumb vs acceleromyography at the big toe. Acta Anaesthesiol Scand 1999; 43: 550-5.

[12] Kern SE, Johnson JO, Orr JA, Westenskow DR. Clinical analysis of the flexor hallucis brevis as an alternative site for monitoring neuromuscular block from mivacurium. J Clin Anesth 1997; 9: 383-7.

[13] Kitajima T, Ishii K, Kobayashi T, Ogata H. Differential effects of vecuronium on the thumb and great toe as measured by accelography and electromyography. Anaesthesia 1995; 50: 76-8.

[14] Sopher MJ, Sears DH, Walts LF. Neuromuscular function monitoring comparing the flexor hallucis brevis and adductor pollicis muscles. Anesthesiology 1988; 69: 129-31.

[15] Saitoh Y, Fujii Y, Takahashi K, Makita K, Tanaka H, Amaha K. Recovery of post-tetanic count and train-of-four responses at the great toe and thumb. Anaesthesia 1998; 53: 244-8. 
[16] Thilen SR, Hansen BE, Ramaiah R, Kent CD, Treggiari MM, Bhananker SM. Intraoperative neuromuscular monitoring site and residual paralysis. Anesthesiology 2013; 117: 964-72.

[17] Lee GC, Lyengar S, Szenohradszky J, et al. Improving the design of muscle relaxant studies: stabilization period and tetanic recruitment. Anesthesiology 1997; 86: 48-54.

[18] Symington MJ, McCoy EP, Mirakhur RK, Kumar N. Duration of stabilization of control responses affects the onset and duration of action of rocuronium but not suxamethonium. Eur J Anaesthesiol 1996; 13: 377-80.

[19] Rimaniol JM, Dhonneur G, Sperry L, Duvaldestin P. A comparison of the neuromuscular blocking effects of atracurium, mivacurium, and vecuronium on the adductor pollicis and the orbicularis oculi muscle in humans. Anesth Analg 1996; 83: 808-13.

[20] Eriksson LI, Sundman E, Olsson R, et al. Functional assessment of the pharynx at rest and during swallowing in partially paralyzed humans: simultaneous videomanometry and mechanomyography of awake human volunteers. Anesthesiology 1997; 87: 1035-43.

[21] Eriksson LI, Sato M, Severinghaus JW. Effect of a vecuroniuminduced partial neuromuscular block on hypoxic ventilatory response. Anesthesiology 1993 ; 78: 693-9.

[22] Ali HH, Wilson RS, Savarese JJ, Kitz RJ. The effect of tubocurarine on indirectly elicited train-of-four muscle response and respiratory measurements in humans. Br J Anaesth 1975; 47: 570-4.

[23] Brand JB, Cullen DJ, Wilson NE, Ali HH. Spontaneous recovery from nondepolarizing neuromuscular blockade: correlation between clinical and evoked responses. Anesth Analg 1977; 56: 55-8.

[24] Engbaek J. Monitoring of neuromuscular transmission by electromyography during anaesthesia. a comparison with mechanomyography in cat and man. Dan Med Bull 1996; 43: 301-16.

(C) Sugi et al.; Licensee Bentham Open.

This is an open access article licensed under the terms of the Creative Commons Attribution Non-Commercial License (http://creativecommons.org/licenses/by-nc/3.0/) which permits unrestricted, non-commercial use, distribution and reproduction in any medium, provided the work is properly cited. 